\title{
Investigating the Current Collapse Mechanisms of p-GaN Gate HEMTs by Different Passivation Dielectrics
}

\author{
Xiangdong Li, Member, IEEE, Niels Posthuma, Benoit Bakeroot, Hu Liang, Shuzhen You, Zhicheng \\ Wu, Ming Zhao, Guido Groeseneken, Fellow, IEEE, and Stefaan Decoutere
}

\begin{abstract}
In this letter, the dynamic $R_{0 n}$ degradation mechanisms of the p-GaN gate HEMTs induced by OFF-state stress are investigated with different passivation dielectrics AION and $\mathrm{SiN}$. The degradation mechanisms are twofold, including $V_{\mathrm{TH}}$ shift and surface trapping in the gate-to-drain access region, whose impacts are successfully distinguished. Surface trapping by $\mathrm{SiN}$ passivation is evidently proved to be the dominant factor that can almost induce a full current collapse. The $V_{\text {TH }}$ positive shift diminishes the drain current by shrinking the overdrive $V_{G S}$, which however can be compensated by a higher $V_{G S}$ overdrive in applications. SiN passivation can effectively suppress the positive bias temperature instability (PBTI) effect, probably by passivating the p-GaN fast traps with hydrogen during passivation. Last, the transient measurements unveil that both the surface trapping and $V_{\mathrm{TH}}$ shift have a very slow recovery process.
\end{abstract}

Index Terms - p-GaN gate HEMTs, dynamic $R_{\mathrm{ON}}$, $V_{\mathrm{TH}}$ shift, surface trapping

\section{INTRODUCTION}

$\mathrm{C}$ OMMERCILIATION of the enhancement-mode (e-mode) p-GaN gate HEMTs has been started recently, especially in the field of consumer electronics such as fast chargers $[1][2][3][4]$. There are mainly two types of e-mode HEMTs available, i.e., Schottky-type p-GaN gate HEMTs [1][2] and hybrid-drain-embedded Gate Injection Transistor (HD-GIT) [3][4]. Reliability issues beyond the JEDEC standard [5] such as current collapse, $\mathrm{p}-\mathrm{GaN}$ gate failure, $V_{\mathrm{TH}}$ shift, etc., have been hampering the wide applications of the GaN power electronic devices.

Current collapse, or dynamic $R_{\mathrm{ON}}$ degradation, after high-voltage OFF-state stress has been heavily investigated for a long time. This problem is normally ascribed to several possible reasons including 1) surface trapping in the gate-to-drain access region $[6][7], 2) V_{\mathrm{TH}}$ positive shift $[8][9][10][11]$, and 3) buffer trapping [12][13][14].For the Schottky-type p-GaN gate HEMTs, we have previously demonstrated p-GaN gate HEMTs with the current collapse

This paragraph of the first footnote will contain the date on which you submitted your paper for review. This work was supported in part by the Vlaams Agenschap Innoveren en Ondernemen and the imec.icon research program.

X. Li, Z. Wu, and G. Groeseneken are with the Department of Electrical Engineering, KU Leuven, 3001 Leuven, Belgium, and also with imec, 3001 Leuven, Belgium (e-mail: Xiangdong.Li@imec.be).

N. Posthuma, H. Liang, S. You, M. Zhao, and S. Decoutere are with imec, 3001 Leuven, Belgium.

B. Bakeroot is with CMST, imec and Ghent University, 9052 Ghent, Belgium. well limited within $\pm 20 \%$ by a bi-layer passivation [15][16]. It has also been unveiled that dedicated buffer stack design and surface passivation can effectively suppress the buffer trapping [14] and surface trapping [6].

Although the surface trapping and $V_{\text {TH }}$ positive shift have been experimentally confirmed to contribute to the current collapse, their impacts are however difficult to be distinguished. Plus, the impact of passivation on the $V_{\mathrm{TH}}$ is seldom reported. In this work, the trapping and de-trapping behaviors will be unambiguously identified on the $\mathrm{p}-\mathrm{GaN}$ gate HEMTs with different passivation dielectrics. Double-pulsed $I-V$ and transient measurements will be conducted to stress and monitor the recovery behavior of the trapping effects.

\section{EPITAXY AND FABRICATION}

The $\mathrm{p}-\mathrm{GaN} / \mathrm{AlGaN} / \mathrm{GaN}$ structure was epitaxially grown by a metalorganic chemical vapor deposition (MOCVD) on 200

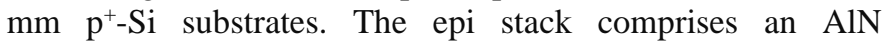
nucleation layer, a superlattice layer, a C:GaN layer, a GaN channel layer, an AlGaN barrier layer, and a Mg-doped p-GaN layer. Detailed processing can be found in [15]. The measured DC and power HEMTs have a gate width $W_{\mathrm{G}}$ of $100 \mu \mathrm{m}$ and 36 $\mathrm{mm}$, respectively. They share the same gate length $L_{\mathrm{G}}$ of $1.5 \mu \mathrm{m}$, gate-source distance $L_{\mathrm{GS}}$ of $0.75 \mu \mathrm{m}$, and gate-drain distance $L_{\mathrm{GD}}$ of $16 \mu \mathrm{m}$. There are four field plates with lengths of $2,4,6$, and $8 \mu \mathrm{m}$, measured from the gate edge of the drain side. Two wafers were compared with different passivation dielectrics of $\mathrm{AlON}$ and $\mathrm{SiN}$ on top of the AlGaN barrier layer. The double-pulsed measurements were performed using an Auriga AU4850 pulsed IV/RF characterization system. The DC and transient recovery measurements were performed by Keysight B1505A. The fast sweeping measurements were conducted by Keysight B1530A WGFMU (Waveform Generator/ Fast Measurement Unit).

\section{Results AND Discussion}

Fig. 1 and Fig. 2 show the $I_{\mathrm{D}}-V_{\mathrm{D}}$ output characteristics and $I_{\mathrm{D}}-V_{\mathrm{G}}$ transfer characteristics of the 100- $\mu \mathrm{m}$ DC HEMTs. The SiN-passivated HEMTs feature a low $V_{\mathrm{TH}}$ of $1.1 \mathrm{~V}$ and a very sharp subthreshold slope, implying the SiN passivation can significantly impact the electrical performance of the $\mathrm{p}-\mathrm{GaN}$ gate HEMTs. AlON-passivated HEMTs resemble the previous reported devices with a high $V_{\mathrm{TH}}>3 \mathrm{~V}$ and a smoother subthreshold slope, ascribed to the trapping effect in the p-GaN layer [17][18]. 

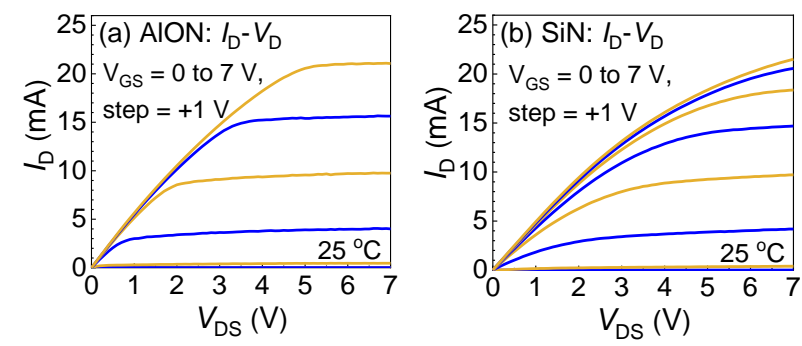

Fig. 1. Output characteristics of the 100- $\mu \mathrm{m}$ (a) AlON-passivated and (b) SiN-passivated HEMTs at $25^{\circ} \mathrm{C}$.
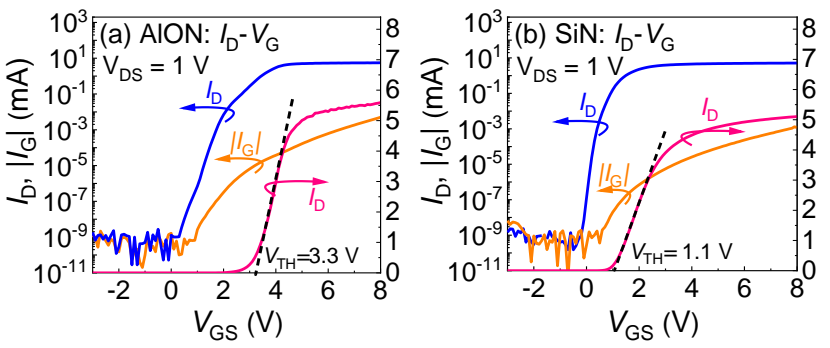

Fig. 2. Transfer characteristics of the 100- $\mu \mathrm{m}$ (a) AlON-passivated and (b) SiN-passivated HEMTs at $25^{\circ} \mathrm{C}$.
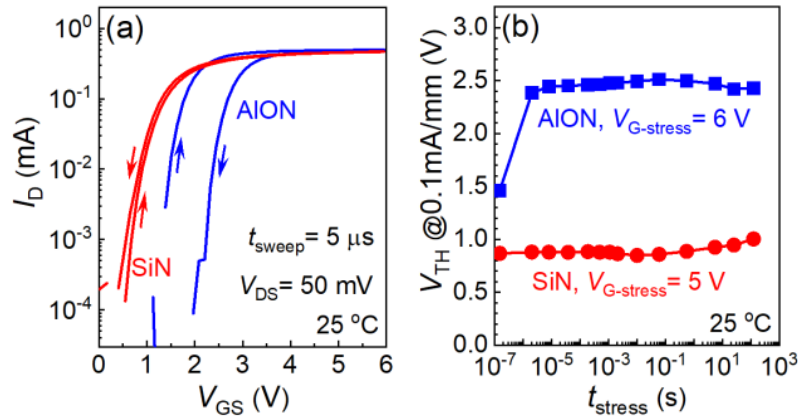

Fig. 3. (a) Double-sweep $I_{\mathrm{D}}-V_{\mathrm{G}}$ curves and (b) $V_{\mathrm{TH}}$ evolution during the forward gate voltage stress of the $100-\mu \mathrm{m}$ AlON-passivated and SiN-passivated HEMTs, by the fast sweeping measurements with an ultra-short $I_{\mathrm{D}}-V_{\mathrm{G}}$ sweep time $t_{\text {sweep }}$ of $5 \mu \mathrm{s}$.

Fast sweeping $I_{\mathrm{D}}-V_{\mathrm{G}}$ curves by B1530A WGFMU are demonstrated in Fig. 3(a). Strikingly, the hysteresis of the SiN-passivated HEMTs is as small as $70 \mathrm{mV}$. Further positive bias temperature instability (PBTI) characterization was conducted by stressing the gate by $6 \mathrm{~V}$ and $5 \mathrm{~V}$ on the AlON-passivated and SiN-passivated HEMTs, respectively. During the characterization, their source terminals were grounded, and the drain terminals were biased at $50 \mathrm{mV}$. It is shown in Fig. 3(b) that the SiN-passivated HEMTs possess a lower but much more stable $V_{\mathrm{TH}}$. This behavior is probably induced by the hydrogen passivation effect, where the hydrogen is introduced during the $\mathrm{SiN}$ deposition. It is worthwhile to mention that only the fast characterization can observe such a significant hysteresis and $V_{\mathrm{TH}}$ instability effect on AlON-passivated HEMTs, because of the p-GaN fast trapping and de-trapping characteristics [17].

To characterize the current collapse, the devices were then subjected to double-pulsed $I_{\mathrm{D}}-V_{\mathrm{D}}$ measurements. The waveforms are depicted in Fig. 4(a). In the OFF state, the devices were stressed by ( $\left.V_{\mathrm{GS} \_\mathrm{Q}}, V_{\mathrm{DS} \_\mathrm{Q}}\right)$, followed by a short dead-time of $200 \mathrm{~ns}$. Afterwards, the devices were turned on by the $\left(V_{\mathrm{GS} \_\mathrm{NQ}}, V_{\mathrm{DS} \_\mathrm{NQ}}\right)$, thus the drain current can be monitored after each OFF-state stress. It is known that the drain current decrease in the $I_{\mathrm{D}}-V_{\mathrm{D}}$ saturation region and linear region is the signature of $V_{\mathrm{TH}}$ shift and trapping in the access region, respectively [19]. Fig. 4(b) and (c) clearly indicate that these two devices both suffer from $V_{\mathrm{TH}}$ shifts. However, only the SiN-passivated HEMTs possess a dramatic trapping in the gate-to-drain access region, leading to a significant drain current collapse.

(a)
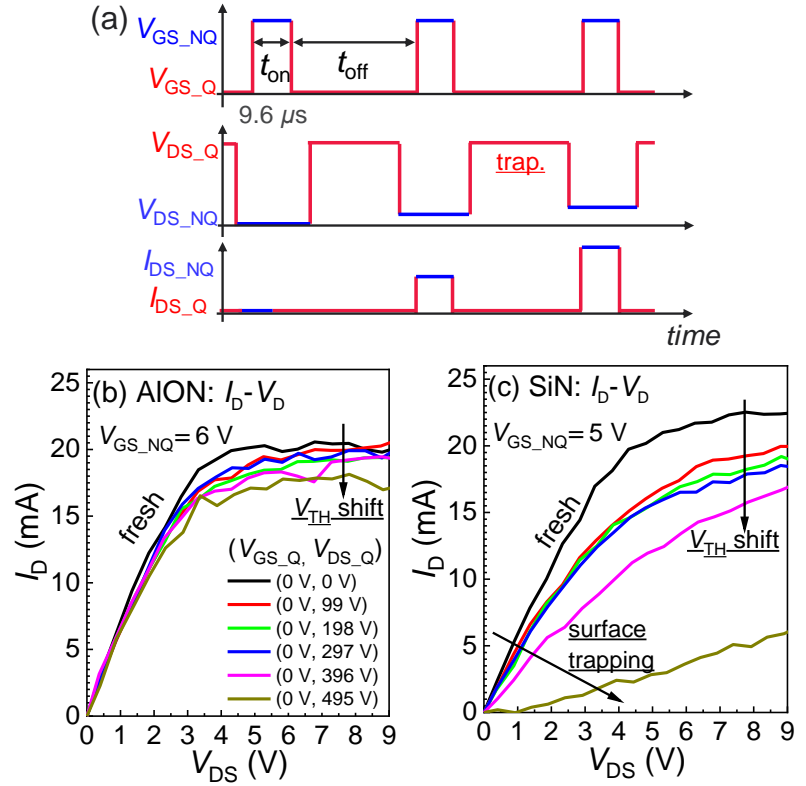

Fig. 4. Double-pulsed $I_{\mathrm{D}}-V_{\mathrm{D}}$ (a) waveforms and $I_{\mathrm{D}}-V_{\mathrm{D}}$ curves for the $100-\mu \mathrm{m}$ (b) AlON-passivated HEMTs and (c) SiN-passivated HEMTs. The quiescent stressing $V_{\text {DS-Q }}$ is $0,99,198,297,396$, and 495 V.
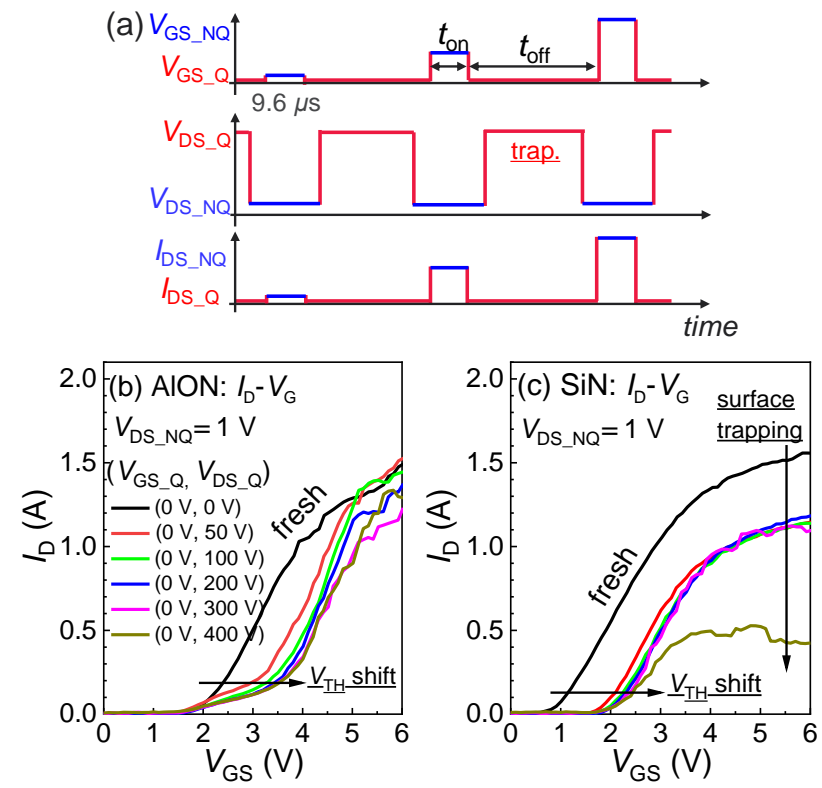

Fig. 5. Double-pulsed $I_{\mathrm{D}}-V_{\mathrm{G}}$ (a) waveforms and $I_{\mathrm{D}}-V_{\mathrm{G}}$ curves for the 36-mm (b) AlON-passivated HEMTs and (c) SiN-passivated HEMTs. The quiescent stressing $V_{\mathrm{DS}-\mathrm{Q}}$ is $0,50,100,200,300$, and $400 \mathrm{~V}$.

Double-pulsed $I_{\mathrm{D}}-V_{\mathrm{G}}$ measurements were further performed 
as shown in Fig. 5. Both devices show a large $V_{\mathrm{TH}}$ shift of more than $1 \mathrm{~V}$. The AlON-passivated HEMTs give an abnormal current bump in the voltage range from 2 to $4 \mathrm{~V}$. This is similar to the results in [17][18], which is possibly induced by trapping under the gate.

Notably, the $I_{\mathrm{D}}$ of the AlON-passivated HEMTs at $V_{\mathrm{GS}}=6 \mathrm{~V}$ under the stress condition of $(0 \mathrm{~V}, 400 \mathrm{~V})$ sees a $20 \%$ decrease because of the positive $V_{\mathrm{TH}}$ shift. Therefore, a large enough $V_{\mathrm{GS}}$ overdrive can effectively compensate the $V_{\mathrm{TH}}$ shift, provided the HEMTs are almost free of surface trapping. This can be verified by the fact that the drain current decreases by $30 \%$ for $V_{\mathrm{GS}}=5 \mathrm{~V}$, whereas $68 \%$ for $V_{\mathrm{GS}}=4 \mathrm{~V}$. The SiN-passivated HEMTs demonstrate a definite evidence of surface trapping, especially under the stress condition of $(0 \mathrm{~V}, 400 \mathrm{~V})$, where the drain current decreases by $65 \%$. This is similar to the excessive current collapse reported in literature of the GIT HEMTs under high-voltage stress [20].

It has been shown in Fig. 3(b) that $\mathrm{SiN}$ passivation can efficiently suppress the PBTI effect. However, the $V_{\mathrm{TH}}$ shift appears again under the OFF-state drain stress. This proves that the OFF-stress-induced $V_{\mathrm{TH}}$ shift possesses a different mechanism from that of the gate PBTI. Previous studies have also pointed out that, instead of the p-GaN trapping effect, the trapping taking place in the $\mathrm{AlGaN}$ barrier [8] and $\mathrm{GaN}$ buffer [9], or p-GaN charging effect [10] are possibly responsible for the OFF-stress-induced $V_{\mathrm{TH}}$ shift.

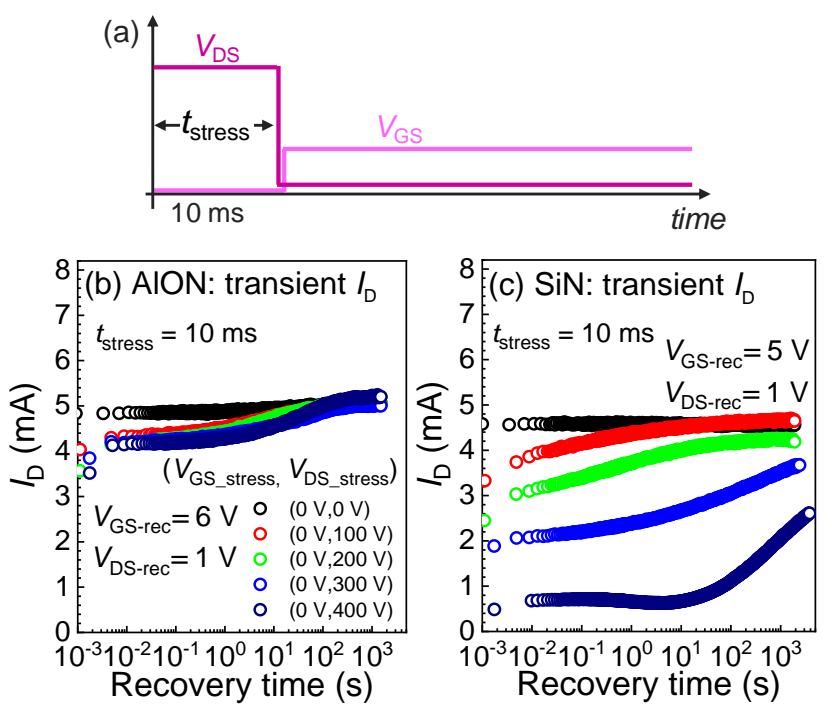

Fig. 6. (a) Stress and sense procedure, and the current transient waveforms of the 100- $\mu \mathrm{m}$ (b) AlON-passivated HEMTs and (c) SiN-passivated HEMTs during the recovery phase. The stress condition is $V_{\mathrm{GS}}=0 \mathrm{~V} / V_{\mathrm{DS}}=400 \mathrm{~V}$ and the stress time is $10 \mathrm{~ms}$.

To monitor the de-trapping behavior, transient measurements were conducted with a Keysight B1505A. As shown in Fig. 6(a), the HEMTs were first subjected to a 10-ms OFF-state stress, then the stress was released, the HEMTs were switched on and the drain current recovery behavior was recorded. There is around 3-ms transition time between the stress and recovery. The AlON-passivated HEMTs show an almost constant current decrease of $13 \%$, irrespective of the stressing voltages. In contrast, the SiN-passivated HEMTs show a monotonous current decrease with increasing stressing voltages.
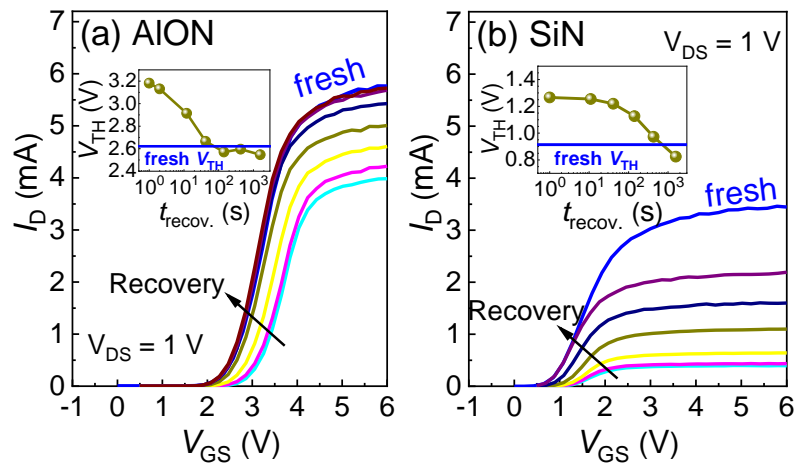

Fig. 7. Transfer $I_{\mathrm{D}}-V_{\mathrm{G}}$ curves of the $100-\mu \mathrm{m}$ (a) AlON-passivated HEMTs and (b) SiN-passivated HEMTs during the recovery phase. The stress condition is $V_{\mathrm{GS}}=0 \mathrm{~V} / V_{\mathrm{DS}}=400 \mathrm{~V}$. The insets show the $V_{\mathrm{TH}}$ evolution during the recovery phase.

The recovery process was periodically interrupted by the $I_{\mathrm{D}}-V_{\mathrm{G}}$ sweeps as shown in Fig. 7. For the SiN-passivated HEMTs, the $V_{\mathrm{TH}}$ and surface trapping were recovering simultaneously. After $1490 \mathrm{~s}$, the $V_{\mathrm{TH}}$ fully recovered, but the surface trapping still existed. For the AlON-passivated HEMTs, the $V_{\mathrm{TH}}$ recovery dominated the current recovery process. Due to the limitation of the setup, the transition between the recovery and sweep takes around $1 \mathrm{~s}$, whose impact is however negligible because of the very slow recovery process.

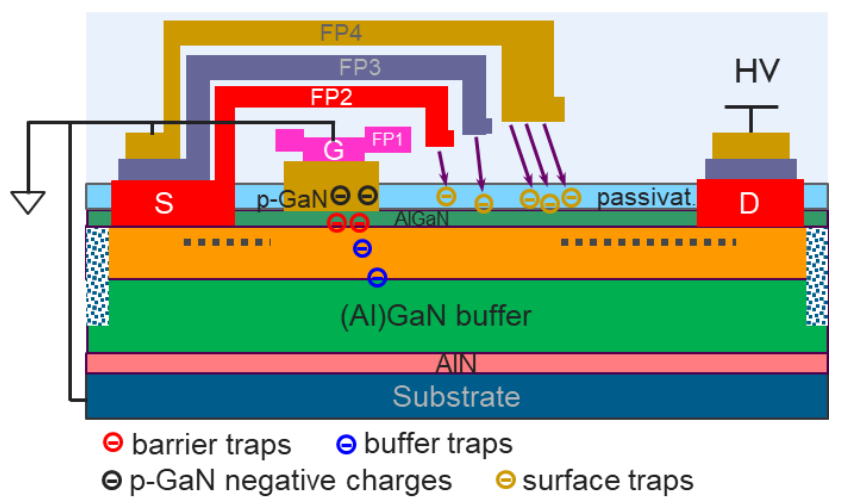

Fig. 8. Schematic cross-section of the $\mathrm{p}-\mathrm{GaN}$ gate HEMTs with four field plates. Possible trapping occurs in the AlGaN barrier, $\mathrm{GaN}$ buffer, and gate-to-drain access region, and negative charging of the $\mathrm{p}-\mathrm{GaN}$ is depicted as well.

Based on the experiments and comparison above, the possible current collapse mechanisms are depicted in Fig. 8. The surface trapping in the gate-to-drain access region is evidently dominant if the passivation quality is imperfect, which can be efficiently suppressed by using the AlON passivation dielectric, or similarly with $\mathrm{Al}_{2} \mathrm{O}_{3}$ in our previous work [15]. Another dominant factor of $V_{\mathrm{TH}}$ shift possibly stems from the trapping effects in the AlGaN barrier and GaN buffer, or from the p-GaN charging effect due to the charged gate-to-drain capacitance $C_{\mathrm{GD}}$. The $V_{\mathrm{TH}}$ shifts induced by the 
OFF-state drain-voltage stress and ON-state gate-voltage stress seem different from each other, because the OFF-stress-induced $V_{\mathrm{TH}}$ shift has a recovery time constant of around 10 to $100 \mathrm{~s}$ (Fig. 7), whereas the PBTI effect time constant is approximately $1 \mathrm{~ms}$ [17].

\section{CONCLUSION}

Current collapse caused by $V_{\mathrm{TH}}$ shift and trapping in the gate-to-drain access region has been successfully distinguished and confirmed by passivating the p-GaN gate HEMTs with different dielectrics $\mathrm{AlON}$ and $\mathrm{SiN}$. The trapping and de-trapping behaviors were comprehensively monitored by various characterization methods such as fast sweeping, double-pulsed $I_{\mathrm{D}}-V_{\mathrm{D}}$ and $I_{\mathrm{D}}-V_{\mathrm{G}}$, and transient measurements. The $V_{\mathrm{TH}}$ shift by OFF-state stress was found to always exist in the Schottky-type p-GaN gate HEMTs, which however can be compensated by a large gate overdrive voltage. The trapping in the access region that induces a severe current collapse was proved to exactly locate in the SiN dielectric. This trapping can induce an almost full current collapse at high OFF-state $V_{\mathrm{DS}}$ of $400 \mathrm{~V}$, which can be effectively suppressed by using the high-quality $\mathrm{AlON}$ or $\mathrm{Al}_{2} \mathrm{O}_{3}$ passivation layer. Both the $V_{\mathrm{TH}}$ recovery and surface de-trapping processes are very slow. Moreover, SiN passivation was found to be able to efficiently suppress the PBTI effect, probably by passivating the $\mathrm{p}-\mathrm{GaN}$ fast traps with hydrogen.

\section{Acknowledgement:}

The authors thank ASM for their contribution on the AlON dielectric layer deposition.

\section{REFERENCE:}

1 Navitas, NV6115 Datasheet. (2018). [Online]. Available:
https://www.navitassemi.com.

2 GaNSystem, GS66504B Datasheet. (2018). [Online]. Available: https://gansystems.com.

3 Infineon, IGT60R190D1S Datasheet. (2020). [Online]. Available: https://www.infineon.com.

4 Panasonic, PGA26E07BA, Datasheet. (2019). [Online]. Available: https://industrial.panasonic.com.

5 Wide Bandgap Power Semiconductors: GaN, SiC. [Online]. Available: https://www.jedec.org/category/technology-focus-area/wide-bandgap-power-s emiconductors-gan-sic.

${ }^{6}$ M. Meneghini, P. Vanmeerbeek, R. Silvestri, S. Dalcanale, A. Banerjee, D. Bisi, E. Zanoni, G. Meneghesso, and P. Moens., "Temperature-dependent dynamic $R_{\mathrm{ON}}$ in GaN-based MIS-HEMTs: Role of surface traps and buffer leakage," IEEE Trans. Electron Devices, vol. 62, no. 3, pp. 782-787, Mar. 2015, doi: 10.1109/TED.2014.2386391.

${ }^{7}$ R. Vetury, N. Q. Zhang, S. Keller, and U. K. Mishra, "The impact of surface states on the DC and RF characteristics of AlGaN/GaN HFETs," IEEE Trans. Electron Devices, vol. 48, no. 3, pp. 560-566, Mar. 2001, doi: 10.1109/16.906451.

${ }^{8}$ L. Efthymiou, K. Murukesan, G. Longobardi, F. Udrea, A. Shibib, and K. Terrill, "Understanding the threshold voltage instability during OFF-state stress in p-GaN HEMTs," IEEE Electron Device Lett., vol. 40, no. 8, pp. 1253-1256, Aug. 2019, doi: 10.1109/LED.2019.2925776.

${ }^{9}$ J. Chen, M. Hua, J. Wei, J. He, C. Wang, Z. Zheng, and K. J. Chen, "OFF-state Drain-voltage-stress-induced $V_{\mathrm{TH}}$ Instability in Schottky-type
p-GaN Gate HEMTs," in IEEE Journal of Emerging and Selected Topics in Power Electronics, doi: 10.1109/JESTPE.2020.3010408.

${ }^{10}$ J. Wei, R. Xie, H. Xu, H. Wang, Y. Wang, M. Hua, K. Zhong, G. Tang, J. $\mathrm{He}$, M. Zhang, and K. J. Chen, "Charge storage mechanism of drain induced dynamic threshold voltage shift in p-GaN gate HEMTs," IEEE Electron Device Lett., vol. 40, no. 4, pp. 526-529, Apr. 2019. doi: 10.1109/LED.2019.2900154.

${ }^{11}$ S. Yang, S. Han, K. Sheng, and K. J. Chen, "Dynamic on-resistance in GaN power devices: Mechanisms, characterizations, and modeling," IEEE J. Emerg. Sel. Topics Power Electron., vol. 7, no. 3, pp. 1425-1439, Sep. 2019, doi: 10.1109/JESTPE.2019.2925117.

12 M. J. Uren, S. Karboyan, I. Chatterjee, A. Pooth, P. Moens, A. Banerjee, and M. Kuball, "'Leaky dielectric' model for the suppression of dynamic $R_{\mathrm{ON}}$ in carbon-doped AlGaN/GaN HEMTs," IEEE Trans. Electron Devices, vol. 64, no. 7, pp. 2826-2834, Jul. 2017, doi: 10.1109/TED.2017.2706090.

${ }^{13}$ S. Yang, C. Zhou, S. Han, J. Wei, K. Sheng, and K. J. Chen, "Impact of substrate bias polarity on buffer-related current collapse in AlGaN/GaNon-Si power devices," IEEE Trans. Electron Devices, vol. 64, no. 12, pp. 5048-5056, Dec. 2017, doi: 10.1109/TED.2017.2764527.

${ }^{14}$ S. Stoffels, M. Zhao, R. Venegas, P. Kandaswamy, S. You, T. Novak, Y. Saripalli, M. Van Hove, and S. Decoutere, "The physical mechanism of dispersion caused by $\mathrm{AlGaN} / \mathrm{GaN}$ buffers on $\mathrm{Si}$ and optimization for low dispersion," in Proc. IEEE Int. Electron Devices Meeting (IEDM), Dec. 2015, pp. 35.4.1-35.4.4, doi: 10.1109/IEDM.2015.7409833.

${ }^{15}$ N. E. Posthuma, S. You, S. Stoffels, H. Liang, M. Zhao, and S. Decoutere, "Gate architecture design for enhancement mode p-GaN gate HEMTs for 200 and 650V applications," in Proc. IEEE 30th Int. Symp. Power Semicond. Devices ICs (ISPSD), May 2018, pp. 188-191, doi: 10.1109/ispsd.2018.8393634

${ }^{16}$ X. Li, N. Amirifar, K. Geens, M. Zhao, W. Guo, H. Liang, S. You, N. Posthuma, B. De Jaeger, S. Stoffels, B. Bakeroot, D. Wellekens, B. Vanhove, T. Cosnier, R. Langer, D. Marcon, G. Groeseneken, and S. Decoutere, "GaN-on-SOI: Monolithically Integrated All-GaN ICs for Power Conversion," in IEEE International Electron Devices Meeting (IEDM), 2019, pp. 4.4.1-4.4.4, doi: 10.1109/IEDM19573.2019.8993572.

${ }^{17}$ X. Li, B. Bakeroot, Z. Wu, N. Amirifar, S. You, N. Posthuma, M. Zhao, H. Liang, G. Groeseneken, and S. Decoutere, "Observation of Dynamic $V_{\mathrm{TH}}$ of p-GaN Gate HEMTs by Fast Sweeping Characterization," IEEE Electron Device Letters, vol. 41, no. 4, pp. 577-580, 2020, doi: 10.1109/LED.2020.2972971.

18 O. Hilt, A. Knauer, F. Brunner, E. Bahat-Treidel, and J. Wurfl, "Normally-off AlGaN/GaN HFET with p-type GaN gate and AlGaN buffer," $6^{\text {th }}$ Int. Conf. Integr. Power Electron. Syst., pp. 1-4, 2010.

${ }^{19}$ D. Bisi, M. Meneghini, M. Van Hove, D. Marcon, S. Stoffels, T. Wu, S. Decoutere, G. Meneghesso, and E. Zanoni, "Trapping mechanisms in GaN-based MIS-HEMTs grown on silicon substrate," Phys. Status Solidi A, vol. 212, no. 5, pp. 1122-1129, May 2015.

${ }^{20}$ K. Tanaka, T. Morita, H. Umeda, S. Kaneko, M. Kuroda, A. Ikoshi, H. Yamagiwa, H. Okita, M. Hikita, M. Yanagihara, Y. Uemoto, S. Takahashi, H. Ueno, H. Ishida, M. Ishida, and T. Ueda, "Suppression of current collapse by hole injection from drain in a normally-off GaN-based hybrid-drain-embedded gate injection transistor," Appl. Phys. Lett., vol. 107, no. 10, pp. 163502-1-163502-4, Oct. 2015, doi: 10.1063/1.4934184. 